\title{
LA-UR-17-25297
}

Approved for public release; distribution is unlimited.

Title:

Author(s):

Intended for:

Issued:
Air-Buoyant Solids

Beaux, Miles Frank II

Web

2017-07-03 
Disclaimer:

Los Alamos National Laboratory, an affirmative action/equal opportunity employer, is operated by the Los Alamos National Security, LLC for the National Nuclear Security Administration of the U.S. Department of Energy under contract DE-AC52-06NA25396. By approving this article, the publisher recognizes that the U.S. Government retains nonexclusive, royalty-free license to publish or reproduce the published form of this contribution, or to allow others to do so, for U.S. Government purposes. Los Alamos National Laboratory requests that the publisher identify this article as work performed under the auspices of the U.S. Department of Energy. Los Alamos National Laboratory strongly supports academic freedom and a researcher's right to publish; as an institution, however, the Laboratory does not endorse the viewpoint of a publication or guarantee its technical correctness. 


\section{Air-Buoyant Solids}

\section{Internet for Everyone with Floating WiFi Hotspots}

\section{BACKGROUND \& MOTIVATION}

Over half the people living on Earth have no access to internet.

WiFi hotspots suspended from helium weather balloons have been proposed, BUT:

- Helium is becoming more expensive/rare.

- Long term deployment of balloons has proven difficult.

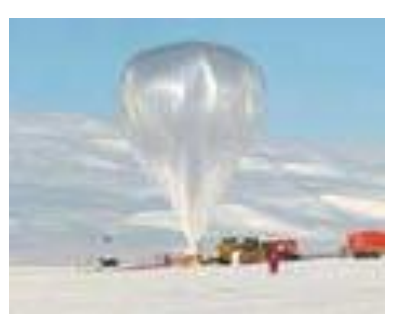

http://asd.gsfc.nasa.gov/

\section{INNOVATION}

Ultra-low density solid supports displacing an evacuated volume

- Advancements in the strength of ultralow density solid materials developed at LANL enables the design of air buoyant solids.

- Hollow geometries that minimize surface area to volume ratio.

- DARPA has developed miniature roughing pumps that can evacuate these "vacuum balloons".

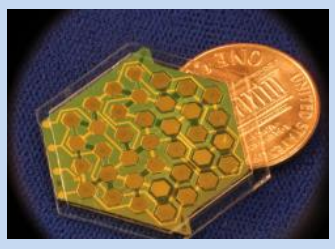

http://newatlas.com/darpa-memssmallest-vacuum-pumps/27883/

\section{DESCRIPTION}

Basically a "Vacuum Balloon", i.e. like a helium weather balloon (or blimp) but requires no gas.

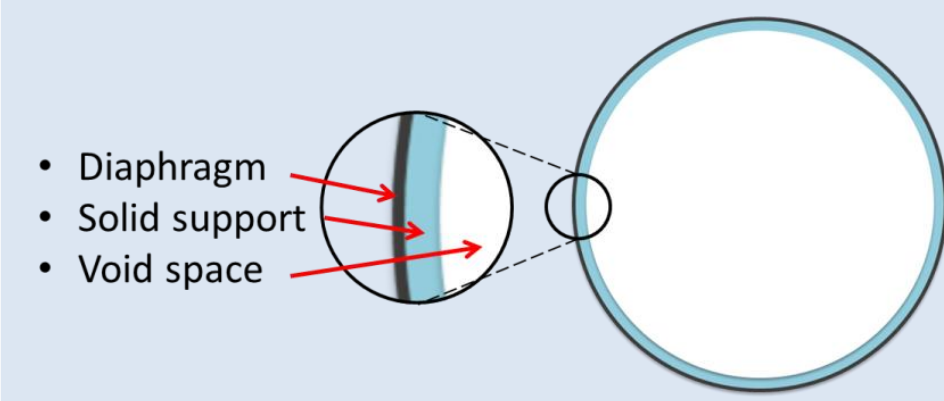

- Instead of filling the balloon with helium, the balloon is instead filled with "nothing".

- Geometry is chosen to minimize surface area to volume ratio, which enables a minimum amount of material to displace a maximum volume of air, thus optimizing buoyancy under evacuation.

- Buoyancy is achieved by pumping air out of the void space inside the solid support shell.

- The solid support shell must withstand atmospheric pressure under evacuation, but be thin enough to achieve buoyancy.

Current Technology Readiness Level (TRL) 3

- Solid support materials have been tested for compression, flow, and vacuum integrity.

- Molds have been produced for making small-scale subbuoyant prototypes for TRL 4 level testing.

- Provisional Patent Pending.

\section{ANTICIPATED IMPACT}

Reduce cost and increase reliability associated with long duration suborbital scientific ballooning

- Reduces much of the expense associated with preventing balloon rupture at altitude.

- More permanent deployment can be achieved.

- Ability to regulate altitude by varying vacuum pressure within structure.

\section{PATH FORWARD}

\section{Provide proof of concept} demonstration with prototype

- Small scale sub-buoyant prototype to determine thickness requirements of solid support.

- Larger scale air-buoyant prototype for proof of concept and demonstration of increased performance with scale up.

- Cost model for mass production.

- TRL increase to level 4; air-buoyant prototype

\section{Potential End Users:}

- NASA, Amazon, Project Loon, academic and private scientific ballooning programs, and other industrial ballooning applications.

Point of Contact: (Miles Beaux, MST-7, (505)695-3241, mbeaux@lanl.gov) 